\title{
The Study of Half-tooth Master Gear Structure Optimization Yazhou Xie ${ }^{a}$, Zhiliang Qian ${ }^{b}$ \\ School of Mechanical and Electric Engineering, Soochow University, China, 215006

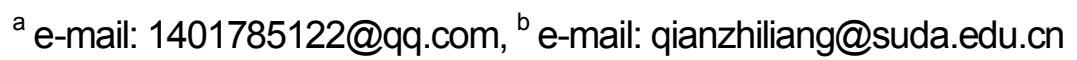

\begin{abstract}
Key words: Half-tooth master gear; Tooth; deformation; Depth of half-tooth groove; Empirical equation Abstract. Aimed at the half-tooth master gear which is used for single and double-flank gear roll testing synchronously. By gear structure analysis, it was discovered that the half-tooth groove depth is the main factor to determine gear tooth stiffness. On the basis, through the finite element analysis of gear with same module and several half-tooth groove depths, the relation between the maximal gear tooth deformation and the half-tooth groove depth was obtained and the optimal half-tooth groove depth corresponding the minimal gear tooth deformation was deduced. Then optimal half-tooth groove depths of several gears with different modules were obtained by the above methods, and an empirical equation was found by which the optimal half-tooth groove depth of a gear can be determined according to its module. Finally, the accuracy and applicability of the empirical equation were verified by example.
\end{abstract}

\section{Introduction}

Single and double-flank gear roll testing are two common gear measuring methods.But measuring principle, measuring elements and measuring results of the two testing are different, and the master gears and measuring instruments used by the two testing are also different, cannot be used interchangeably. Half-tooth master gear is an original gear with distinctive structure [1]. Two half-tooth master gears can make up a variable-tooth-thickness master gears by which single and double-flank gear roll can be tested synchronously, and radial composite deviation and tangential composite deviations of both tooth flanks can be obtained with an instrument and one measuring process[1,2]. This article studied structure optimization of half-tooth master gear to improve its stiffness and applicability.

\section{Tooth Stiffness of the Half-tooth Master Gear}

Structure of the Half-tooth MasterGgear . Half-tooth master gear(Fig. 1) has following features: it only preserve half of traditional gear tooth on the cross section, the other half and partial gear body are cut off to form half-tooth groove; it only preserve half of the traditional gear's spoke and hub on the axial section, the half on the other side of axial symmetric plane is cut off to form a cavity. Obviously, tooth of half-tooth master gear is cantilever-like, its stiffness is evidently weaker than the traditional gear tooth.

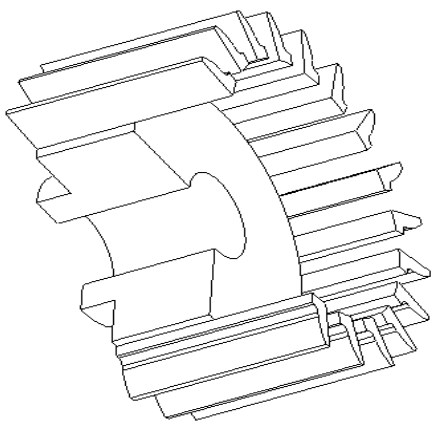

Fig.1.Half-tooth master gear

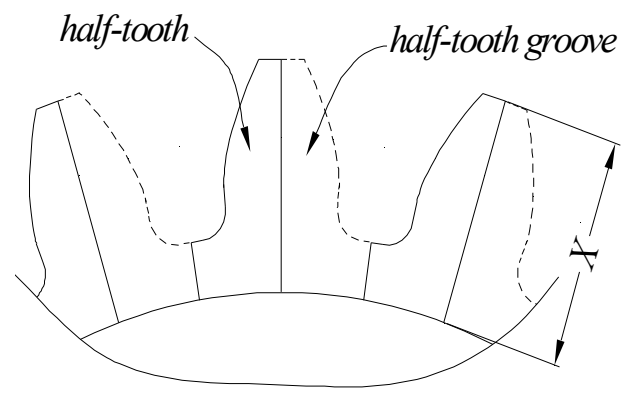

Fig.2. Depth of half-tooth groove $X$

Factors Influencing Tooth Stiffness.Because a variable-tooth-thickness master gears are comprised of two half-tooth master gears, the spoke and hub of half-tooth master gaer on one side of axial symmetric plane must be cut off to form a cavity. That means tooth of half-tooth master gear mst be cantilever-like in half a tooth width along axial direction. Thus, the depth of half-tooth groove $\mathrm{X}$ is the variable structure parameter only (Fig. 2).

Obviously, the cross-sections of tooth overhung end and half-tooth groove on a half-tooth master gear are 
identical in shape. With other conditions being same, the depth of half-tooth groove $\mathrm{X}$ directly decides the shape and size of gear tooth overhung end, and sequentially it will directly affect the gear tooth stiffness.

Influence of Tooth Deformation.Under the same loading condition, the weaker the gear stiffness, the more serious the deformation of gear tooth [3]. In the process of single and double-flank gear roll testing synchronously, the load deformation of master gear tooth can be transmitted to tangential and radial composite deviation s of product gear, and directly affects the accuracy of measuring result.

\section{Analysis Method of Tooth Deformation}

Analysis Method of Tooth Deformation. Computing gear tooth stiffness, gear tooth is usually simplified as variable cross-section cantilever beam. However, half-tooth master gear tooth is cantilevered along radial and axial directions, the problem of its stiffness is far more complicated than the traditional gear tooth's. The computing method of traditional gear tooth stiffness can't be applied here. In view of that the functions of CAE software have been quite perfect and the accuracy of its analysis results has also been verified by lots of engineering examples. The half-tooth master gear tooth stiffness is analyzed by ANSYS workbench in this article. The specific methods are as follows:

1) Under the same constraint and load conditions, the half-tooth master gears with different depths of half-tooth groove are analyzed by ANSYS workbench, and tooth deformation values of gears with different depths of half-tooth groove are obtained.

2) The discrete deformation data are fitted and analyzed to obtain the optimal half-tooth groove depth $X_{m}$ with which the gear tooth deformation is minimum.

3) Repeat steps 1) and 2) for the half-tooth master gears with different modules tp find out the relationship between $\mathrm{X}_{\mathrm{m}}$ and gear module $\mathrm{M}$.

Settings of Gear Deformation Analysis.The deformation of half-tooth master gear is affected not only by gear structure, but also by gear material, constraints and loads, and so on. In this article, the half-tooth master gear tooth deformation is analyzed by ANSYS workbench under following specific settings::

1) Gear material and material properties. The half-tooth master gear belongs to high-precision and wearable master gears, 38CrMoAlA is selected as gear material according to Gear Design Manual[4], elasticity modules and Poisson's ratio are set respectively as $2.09 \times 105 \mathrm{~N} / \mathrm{mm} 2$ and 0.3 . After gear material is selected, physical properties of material can be inherited directly by the solid model of the component.

2) Mapped Meshing. In order to obtain the higher precision of analysis and consider the computation speed, set the mapped meshing size as $2 \mathrm{~mm}$ and the element as tetrahedron unit in this article.

3) Constraint Condition. Restrain six freedom degrees of the axle hole on the half-tooth master gear. Set the constraint of the axis hole as fixed support.

4) Contact Load. During the roll testing of master gear and product gear, the contact load acted on gearing flanks only needs to overcome the friction in supporting shafts and gearing flanks, the specific load value is relevant to the structure of the gear roll testing instrument. On the other hand, the article studies the influence of half-tooth master gear structure on gear tooth stiffness through analyzing the deformation of gears with different depth of half-tooth groove under the same contact load. In the elastic range, the magnitude of load can change the value of tooth deformation, can't change the relation between tooth deformation and gear structure. Thus, the article sets contact load as $10 \mathrm{~N}$ in the following deformation analyses,.

5) Acting Location and Acting Mode of Load. In the deformation analyses, the contact force is set as concentrated load and it is acted at the top point of tooth overhung end, the acting direction of force is perpendicular to tooth flank. During gear roll testing, the root defect of product gear tooth end(for example, convex burr) will produce the above loading effect on master gear. Meanwhile, this is the worst loading situation for the deformation of half-tooth master gear .

\section{The Relationship Between Module and Optimal Depth of Half-tooth Groove}

The half-tooth master gear posesses particular structure(Fig. 1), its tooth is overhung along both axial and radial directions, and the cantilever length $\mathrm{L}$ along radial direction is equal to the depth of half-tooth groove $\mathrm{X}$ (Fig. 2). The longer the depth of half-tooth groove $\mathrm{X}$, the larger the cross section area of overhung gear tooth 
A, and the longer the cantilever length L (Fig. 3). Obviously, the increase of A can improve the stiffness of tooth overhung end, and the increase of $\mathrm{L}$ will reduce rasial bending stiffness of gear tooth. Giving consideration to both, there is an optimal depth of half-tooth groove $\mathrm{X}_{\mathrm{m}}$ which will make the gear deformation minimal at the same load. Namely, it will make the gear tooth stiffness (the overhung end of gear tooth) maximal. Then, this article studies the relationship between module $\mathrm{M}$ and the optimal depth of half-tooth groove $\mathrm{X}_{\mathrm{m}}$ with the above analysis methods.
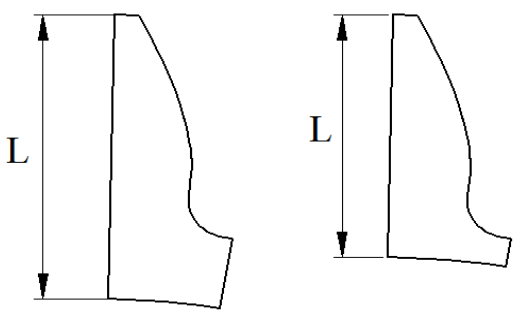

Fig.3.The radial cantilever length $L$
Table 1 The maximal deformationY

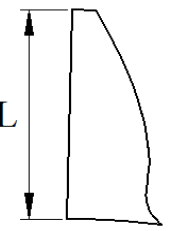

\begin{tabular}{cc}
\hline $\mathrm{X}[\mathrm{mm}]$ & $\mathrm{Y}[\mu \mathrm{m}]$ \\
\hline 8 & 38.065 \\
10 & 24.753 \\
12 & 19.712 \\
14 & 17.696 \\
16 & 16.945 \\
18 & 17.077 \\
20 & 17.758 \\
22 & 19.033 \\
24 & 21.044 \\
\hline
\end{tabular}

The Optimal Depth of Half-tooth Groove underSpecific Module. For the half-tooth master gear of which module $\mathrm{M}$, pressure angle, number of teeth, addendum coefficient and tooth width respectively equals to $3[\mathrm{~mm}], 20^{\circ}, 30,1.0$ and $60[\mathrm{~mm}]$, the article establishes the nine models with nine different depth of half-tooth groove $\mathrm{X}_{\mathrm{i}}$, and analyzes the maximal comprehensive deformation of the tooth overhung end by ANSYS workbench. The maximal comprehensive deformation $\mathrm{Y}$ of tooth overhung end related to the different depth of half-tooth groove $\mathrm{X}$ is shown in Table 1.

The data in Table 1 are fitted according to the least square sextic polynomial fitting by Matlab., The fitting equation and the relationship curve between the depth of half-tooth groove $\mathrm{X}$ and the maximal comprehensive deformation $Y$ are obtained and respectively shown as Eq.1 and Fig.4.

$$
\begin{aligned}
Y(X= & 0.000018106 X^{6}-0.0019263 X^{5}+0.084918 X^{4} \\
& -1.9889 X^{3}+26.2370 X^{2}-186.2500 X+577.8600
\end{aligned}
$$

It is obvious in Fig.5 that there is an optimal depth of half-tooth groove $X_{m}$ with which the maximal tooth deformation is minimal. This article analyzes Eq. 1 is analyzed by Matlab and the optimal depth of half-tooth groove $X_{m}$ is obtained. The optimal depth $\mathrm{Xm}$ is equal to $16.713[\mathrm{~mm}]$.

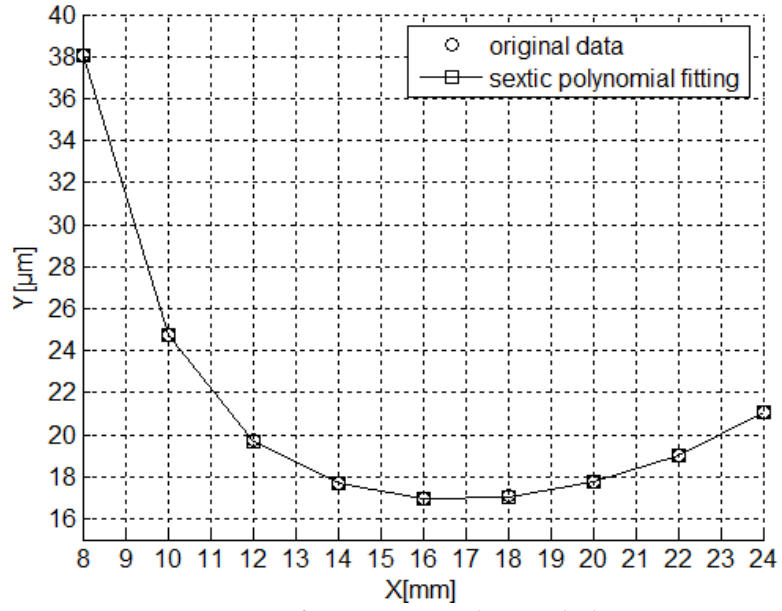

Fig.4 Y\&X for gear with module 3[mm]

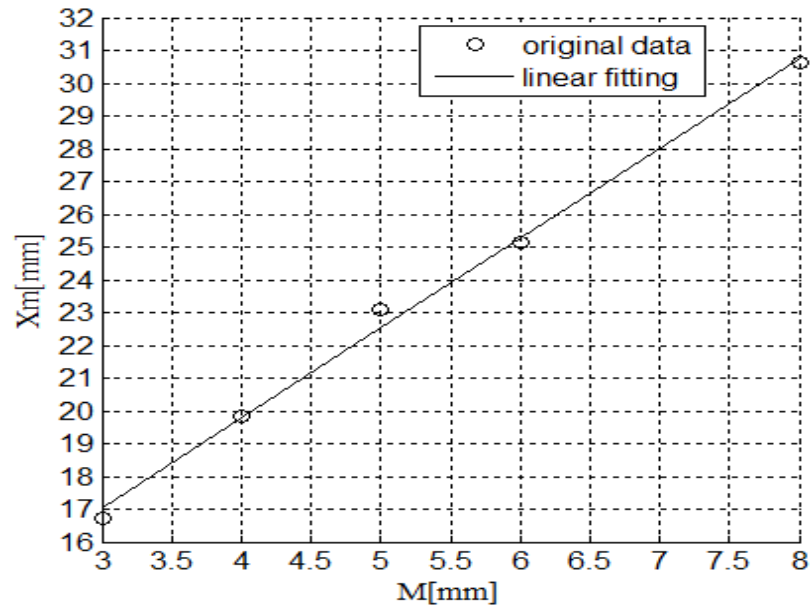

Fig.5 Relationship between $X_{m}$ and $M$ 
Optimal Depth of Half-tooth Groove under Different Module.

Maintaining the above parameters except module unchanged, the article analyzes the half-tooth master gaers of which module is respectively equal to $4,5,6$,and $8[\mathrm{~mm}]$ and obtains the optimal half-tooth depths of the gaers. The optimal depths of gears with different module is shown in Table 2 ..

\section{The Relationship Between Module and the Optimal Depth.}

In order to reveal the relationship between module $M$ and the optimal depth of half-tooth groove $X_{m}$, this article fits the optimal half-tooth groove depths $\mathrm{X}_{\mathrm{m}}$ related to modules $3,4,5,6$ and $8[\mathrm{~mm}]$ with the least square linear fitting. The fitted relationship between module and the optimal half-tooth groove depth is shown as Fig.5. The fitted equation is as following:

$$
X_{m}=2.7521 M+8.7711
$$

There is the good linear relationship between module and the optimal half-tooth groove depth as shown in Fig.5. Eq. 2 has fitting degree up to 0.9959 and can be used as the empirical equation to determine the depth of half-tooth groove. Utilizing the equation, the approximate optimal depth of half-tooth groove $\mathrm{X}_{\mathrm{m}}$, with which the half-tooth master gear will posess approximatively maximum stiffness, can be directly determined according to the gear module $\mathrm{M}$.

\section{Application Example and Verification}

Design a half-tooth master gear with the objective of maximum tooth stiffness, the module of gear is $7[\mathrm{~mm}]$, other parameters are the same as the above.

Design According to the Empirical Equation. Substitute $M=7[\mathrm{~mm}]$ into Eq.2, and abtains the approximate optimal half-tooth groove depth $\mathrm{X}_{\mathrm{ma}}: X_{m a}=2.7521 M+8.7711=2.7521 \times 7+8.7711=28.0358[\mathrm{~mm}]$.

Design According to above AnalysisMethod. With the half-tooth groove depth $\mathrm{X}$ being respectively equal to $20,22,24,26,28,30,32,34$ and $36[\mathrm{~mm}]$, the models are built and analyzed according to above analysis method, and the maximal comprehensive deformation $\mathrm{Y}$ under different half-tooth groove depth $\mathrm{X}$ are obtained as shown in Table 3.

It can be seen from Table 3 that the gear deformation is minimal when the depth of half-tooth groove $\mathrm{X}$ is $28[\mathrm{~mm}]$, which is accordant to the approximate optimal half-tooth groove depth $\mathrm{X}_{\mathrm{ma}}$ obtained by Eq.2. Besides, fitting the data in Table 3 and evaluating its optimal value following above methods, it is obtained that the optimal depth of half-tooth groove $\mathrm{X}_{\mathrm{m}}$ equals $27.407[\mathrm{~mm}]$, which is very close to the optimal depth of half-tooth groove $\mathrm{X}_{\mathrm{ma}}$ obtained from Eq.2.

\section{Conclusion}

Through the finite element analysis of the gear with same module and different half-tooth groove depth, the variation of the maximal gear tooth deformation with the half-tooth groove depth is obtained. Moreover the optimal half-tooth groove depth under which the gear tooth deformation will be minimal is deduced. And then, following the above analysis methods, several optimal half-tooth groove depths of several gears with different module are also obtained. From this, an empirical equation is established through the least square method linear fitting, the optimal half-tooth groove depth can be determined according to gear module by above equation. At last, the usage of this empirical equation is given, and the accuracy and applicability of the equation are also verified by an example. 


\section{References}

1. A Type of Measuring Gear \& Measuring Method, ZL201210255341.4, 2014-12-01

2. Xinmiao Yu, Zhiliang, Qian, et al. New Kind of Master Gear and Measurement Principle for Gear Composite Deviations[J], New Technology \& New Process, 2013.7:20-22

3. Xiongxiong Lu, Zhiliang Qian, et al. Design and Stiffness Verification of a New Type of Measuring Gear[J], New Technology \& New Process, 2013.7:53-56

4. Xiaolu Zhu. Gear Design Manual(second edition). Chemical Industry Press,2010 